\title{
Long-range excitons in conjugated polymers with ring torsions
}

\author{
Kikuo Harigaya周 \\ Physical Science Division, Electrotechnical Laboratory, \\ Umezono 1-1-4, Tsukuba, Ibaraki 305, Japan \\ (Received \\ )
}

\begin{abstract}
Ring torsion effects on optical excitation properties in poly(para-phenylene) (PPP) and polyaniline (PAN) are investigated by the intermediate exciton formalism. Long-range excitons are characterized, and the long-range component of the oscillator strengths is calculated. We find that ring torsions affect the long-range excitons in PAN more easily than in PPP, due to the larger torsion angle of PAN and the large number of bonds whose hopping integrals are modulated by torsions.
\end{abstract}

*E-mail address: harigaya@etl.go.jp; URL: http://www.etl.go.jp/People/harigaya/ 


\section{Introduction}

Recently, we have been studying structures of photoexcited states in electroluminescent conjugated polymers: poly(para-phenylene) (PPP), poly(para-phenylenevinylene) (PPV), poly(paraphenylenedivinylene) (PPD), and so on [1-3]. We have introduced the viewpoint of long-range excitons in order to characterize photoexcited states where an excited electron-hole pair is separated over a single monomer of the polymer. We have shown $[1,2]$ that a long-range exciton feature starts at the energy in the higher energy side of the lowest feature of the optical absorption of PPV. The presence of the photoexcited states with large exciton radius is essential in mechanisms of the strong photocurrents observed in this polymer. In ref. [3], we have compared properties of excitons in PPV-related polymers. The oscillator strengths of the long-range excitons in PPP are smaller than in PPV, and those of PPD are larger than in PPV. Such relative variation is due to the difference of the number of vinylene bonds.

It is known that the PPV chain is nearly planer and the phenyl rings are not distorted each other, as observed in X-ray analysis [4]. However, ring torsions, where even number phenyl rings are rotated in the right direction around the polymer axis and odd number phenyl rings are rotated in the left direction, have been observed in PPP [5]. The polymer structure of PPP is shown schematically in Fig. 1 (a). The ring torsions originate from the steric repulsion between phenyl rings, because the distance between the neighboring rings in PPP is smaller than that of PPV. In a simple tight binding model, the ring torsion modulates the nearest neighbor hopping integral $t$ as $t \cos \Psi$, where $\Psi$ is the torsion angle. If the angle $\Psi$ is sufficiently large, the motion of electrons between phenyl rings would be hindered and also the exciton radius of photoexcited states would become shorter. The contribution from long-range excitons will be smaller from that of the calculations [3] where the planer structure of PPP has been assumed. The actual torsion angle about $23^{\circ}$ [5] may influence the results of the previous calculations. The first purpose of this paper is to examine ring torsion effects on optical excitations of PPP. We will show that the magnitude of the torsion $\Psi \sim 23^{\circ}$ does not change the component of the 
long-range excitons so much.

The another example of polymers where ring torsions are present is polyaniline (PAN) (leucoemeraldine base). The schematic structure is displayed in Fig. 1 (b). The phenyl rings and NH units are arrayed alternatively in the chain direction. The magnitude of the torsion of phenyl rings in PAN is about $56^{\circ}[6]$. This is larger than that of PPP, and thus the photoexcited states in PAN will be influenced more strongly by the torsion. In the last half of this paper, we shall look at this problem. We will show that the long-range component of the oscillator strengths at $\Psi=56^{\circ}$ is about half of the magnitude of the system without ring torsions. Longrange excitons in PAN are hindered by ring torsions more easily than those in PPP. This is due to the fact that the torsion angle is larger in PAN, and also that two bonds, whose hopping integrals are modulated as $t \cos \Psi$, are present between neighboring phenyl rings in PAN while one such bond is present between phenyls in PPP.

This paper is organized as follows. In the next section, ring torsion effects on photoexcited states in PPP are reported. The results of PAN are given in $\S 3$, and the paper is concluded with a summary in $\S 4$.

\section{Ring torsion effects in PPP}

The model used in [3] is modified in order to take account of ring torsions. The Hamiltonian in [3] is composed of the tight binding part and the term of long-range Coulomb interactions among electrons. In the tight-binding part, the nearest neighbor hopping integral is modulated linearly with respect to the bond length change by the electron-phonon coupling $\alpha$. In the term of Coulomb interactions, the parametrized Ohno potential is used among all the carbon sites in order to describe exciton effects. The form of the Ohno potential is $1 / \sqrt{(1 / U)^{2}+(r / a V)^{2}}$, where $U$ and $V$ are the strengths of the onsite and long-range interactions. We have assumed the following parameters in [3]: the mean bond length $a=1.4 \AA, \alpha=2.59 t / \AA$, the harmonic spring constant $K=26.6 t / \AA^{2}, U=2.5 t$, and $V=1.3 t$ ( $t$ is the nearest neighbor hopping 
integral of the system with the equal bond length $a$ ).

In this section, the torsion angle between neighboring phenyl rings $\Psi$ is taken into account in the hopping integral without ring torsions as $t-\alpha y \Rightarrow(t-\alpha y) \cos \Psi$ in the model reviewed above. Here, $y$ is the length change of the corresponding bond. The model is solved by the mean field approximation and electron-hole excitations are calculated by the intermediate exciton formalism. The long-range component of photoexcited states are characterized as we have done in the previous paper [3].

Figure 2 shows the optical absorption spectra calculated for the system with the torsion angle $\Psi=23^{\circ}[5]$ and the monomer number $N_{\mathrm{m}}=20$. The spectral shapes are nearly independent of the monomer number at $N_{\mathrm{m}}=20$, as reported in [3]. The polymer without ring torsions with the open boundary is in the $x-z$ plane. The electric field of light is parallel to the chain and in the direction of the $x$-axis in Fig. 2 (a), and it is perpendicular to the chain and is along with the $y$-axis in Fig. 2 (b). The electric field is along with the $z$-axis in Fig. 2 (c). The orientationally averaged spectra with respect to the electric field are shown in Fig. 2 (d). In each figure, the bold line shows the total absorption and the thin line shows the contribution from the long-range excitons where photoexcited electron and hole are separated over more than the spatial extent of the single monomer. In Fig. 2 (a), there are two main features around $1.4 t$ and 2.4t, where quantities with energy dimensions are expressed in units of $t$. There is a feature of long-range excitons around $\sim 2.0 t$ at the higher energy side of the 1.4t main peak. In contrast, the $2.4 t$ feature does not have so strong long-range component due to the almost localized nature of excitons. This is similar to the calculations in [3], and the ring torsions do not change the almost localized character of the feature around $2.4 t[7]$. Figure 2 (b) shows the case where the electric field is parallel to the $y$-axis. If the ring torsions are not present, the oscillator strengths are zero in this case. However, they are finite and the maximum of the bold line in Fig. 2 (b) is one order of magnitudes smaller than that of Fig. 2 (a). Two main features of the energies of $2.2 t$ and $2.8 t$ are derived from those of the case with the electric field in the $z$-direction, shown in Fig. 2 (c). These features in Fig. 2 (c) contribute dominantly, and 
their oscillator strengths are of the same order as those of Fig. 2 (a). There are long-range components of these two features around the energies about $2.5 t$ and 3.3t. Figure 2 (d) shows

the optical spectra where the electric field is orientationally averaged. The overall spectral shape is similar to that in the case without ring torsions $\Psi=0[3]$. However, the spectral width decreases slightly due to the smaller hopping interactions between neighboring phenyl rings.

Next, we vary the torsion angle $\Psi$ in the Hamiltonian, and look at changes in optical spectra. We calculate the long-range component in the total oscillator strengths, as we have done in [1-3]. We search for its variations as a function of $\Psi$. Figure 3 shows the calculated results. The closed squares are the data of the orientationally averaged absorption. The open circles, squares, and triangles indicate the data for the cases with the electric field parallel to the $x-, y-$, and $z$-axes, respectively. We find that the average long-range component weakly depends on $\Psi$, while $\Psi$ is as large as about $40^{\circ}$. Thus, we have examined ring torsion effects on optical excitations of PPP. We have found that the magnitude of the torsion $\Psi \sim 23^{\circ}$ does not change the component of the long-range excitons so much from that of the torsion free system. However, the long-range component suddenly deceases at larger $\Psi$, and becomes zero at $\Psi=90^{\circ}$. This is a natural conclusion of the broken conjugations due to strong ring torsions. The long-range components with the field in the $y$ - and $z$-directions are generally larger than that of the $x$-direction case. This property has been seen in ref. [3], too.

\section{Ring torsion effects in PAN}

It is of some interests to investigate ring torsion effects in conjugated polymers where torsion angles are larger than that of PPP. In this section, we look at torsion effects in PAN as a typical example of such the polymers. The polymer structure is shown schematically in Fig. 1 (b). The zigzag geometry of PAN is taken into account in the actual calculations, even though it is not shown in Fig. 1 (b). For model parameters, we have assumed the negative site energy 
$E_{\mathrm{N}}=-0.571 t$ at the $\mathrm{N}$ sites, the hopping integral $t_{\mathrm{C}-\mathrm{N}}=0.8 t$ between carbon and nitrogen, the onsite interaction strength $U=2 t$, and the magnitude of long-range interactions $V=1 t$. The parameters of $E_{\mathrm{N}}$ and $t_{\mathrm{C}-\mathrm{N}}$ are taken from [8] as representative values. We show the results with the monomer number $N_{\mathrm{m}}=20$. We have taken several combinations of Coulomb interaction parameters and have looked at changes of results. We have found that the the long-range component as a function of $\Psi$ depends weakly on $U$ and $V$. Thus, we report the results for one combination of Coulomb parameters.

Figure 4 show the calculated optical absorption spectra of PAN with the torsion angle $\Psi=56^{\circ}$. The three dimensional coordinates of the polymer are similar to those of PPP in Fig. 2. In Fig. 4 (a), the electric field is along with the polymer axis, and is in the $x$-direction. There are two main features around $0.6 t$ and 2.6t. The former has smaller long-range exciton feature than the latter. As we observe in the band structure of PAN [8], the lowest optical excitation among the band gap has a certain magnitude of dispersions, and thus its long-range component might be observed. However, there is the second lowest optical excitation, which is almost localized, at the excitation energy just above the lowest exciton. Thus, the long-range component of the $0.6 t$ feature is suppressed. Figure 4 (b) shows the weak optical absorption originated from the finite torsions as we have found for the PPP in Fig. 2 (b). Figure 4 (c) shows the optical spectra when the electric field is in the $z$-direction. Two features around $1.2 t$ and 2.6t have certain magnitudes of oscillator strengths due to long-range excitons. This qualitative property is similar to that in Fig. 2 (c). Then, Fig. 4 (d) shows the optical spectra where orientational average is performed. We find excitation features superposed from those of Figs. 4 (a), (b), and (c).

Finally, we show the long-range component of the oscillator strength as a function of the torsion angle $\Psi$ in Fig. 5. In contrast to PPP, the long-range component decreases smoothly as $\Psi$ increases. The long-range component of the total oscillator strengths at the observed $\Psi=56^{\circ}$ is about half of the magnitude of the system without ring torsions. Thus, the longrange excitons in PAN can be hindered by ring torsions more easily than those in PPP. This is 
due to the fact that the torsion angle is larger in PAN, and also that two bonds, whose hopping integrals are modulated as $t \cos \Psi$, are present between neighboring phenyl rings in PAN while one such kind of bond is present between phenyls in PPP.

\section{Summary}

We have examined the ring torsion effects which might interrupt delocalizations of excitons in the chain direction of the conjugated polymers PPP and PAN. Long-range excitons in the optical excitations have been characterized, and the long-range component of oscillator strengths is calculated as a function of the torsion angle. We have shown that the torsion effects in PPP are relatively small. In contrast, the torsions of PAN decrease the long-range component by about half of the magnitudes from that of the torsion-free system.

\section{Acknowledgements}

Useful discussion with Y. Shimoi, S. Abe, and K. Murata is acknowledged. Numerical calculations have been performed on the DEC AlphaServer of Research Information Processing System Center (RIPS), Agency of Industrial Science and Technology (AIST), Japan. 


\section{References}

[1] K. Harigaya, J. Phys. Soc. Jpn. 66, to be published in No. 5 issue (1997).

[2] K. Harigaya, J. Phys.: Condens. Matter 9, to be published in July issue (1997).

[3] K. Harigaya, J. Phys.: Condens. Matter 9, to be published (1997).

[4] D. Chen, M. J. Winokur, M. A. Masse, and F. E. Karasz, Phys. Rev. B 41, 6759 (1990).

[5] J. L. Baudour, Y. Delugeard, and P. Rivet, Acta Crystallogr. B 34, 625 (1978).

[6] M. E. Jozefowicz, R. Laversanne, H. H. S. Javadi, A. J. Epstein, J. P. Pouget, X. Tang, and A. G. MacDiarmid, Phys. Rev. B 39, 12958 (1989).

[7] Z. G. Soos, S. Etemad, D. S. Galvão, and S. Ramasessha, Chem. Phys. Lett. 194, 341 (1992).

[8] J. M. Ginder and A. J. Epstein, Phys. Rev. B 41, 10674 (1990). 


\section{Figure Captions}

Fig. 1. Polymer structures of (a) PPP and (b) PAN. These figures are only schematic. Geometries with ring torsions and the zigzag chain structure of PAN are used in the actual calculations.

Fig. 2. Optical absorption spectra of the PPP shown in arbitrary units. The polymer axis is parallel to the $x$-axis. The polymer without ring torsions is in the $x$ - $z$ plane. The electric field of light is parallel to the chain and in the direction of the $x$-axis in (a), and it is perpendicular to the chain and is along with the $y$-axis in (b). It is along with the $z$-axis in (c). The orientationally averaged spectra are shown in (d). The number of the PPP monomer units is $N_{\mathrm{m}}=20$. The bold line is for the total absorption. The thin line indicates the absorption of the long-range component. The Lorentzian broadening $\gamma=0.15 t$ is used.

Fig. 3. Long-range component of the optical absorption spectra as a function of the torsion angle in PPP. The monomer unit number is $N_{\mathrm{m}}=20$. The closed squares are for the orientationally averaged absorption. The open circles, squares, and triangles indicate the data for the cases with the electric field parallel to the $x-, y$-, and $z$-axes, respectively.

Fig. 4. Optical absorption spectra of the PAN shown in arbitrary units. The polymer axis is parallel to the $x$-axis. The polymer without ring torsions is in the $x$ - $z$ plane. The electric field of light is parallel to the chain and in the direction of the $x$-axis in (a), and it is perpendicular to the chain and is along with the $y$-axis in (b). It is along with the $z$-axis in (c). The orientationally averaged spectra are shown in $(\mathrm{d})$. The number of the PAN monomer units is $N_{\mathrm{m}}=20$. The bold line is for the total absorption. The thin line indicates the absorption of the long-range component. The Lorentzian broadening $\gamma=0.15 t$ is used.

Fig. 5. Long-range component of the optical absorption spectra as a function of the torsion 
angle in PAN. The monomer unit number is $N_{\mathrm{m}}=20$. The closed squares are for the orientationally averaged absorption. The open circles, squares, and triangles indicate the data for the cases with the electric field parallel to the $x^{-}, y$-, and $z$-axes, respectively. 
(a) PPP

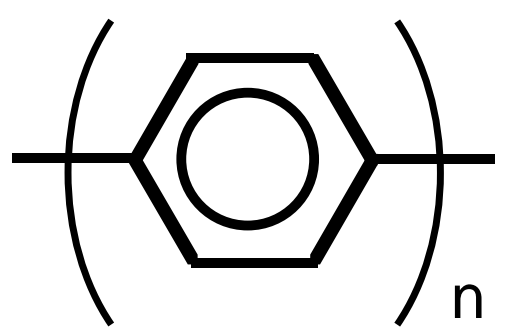

(b) PAN

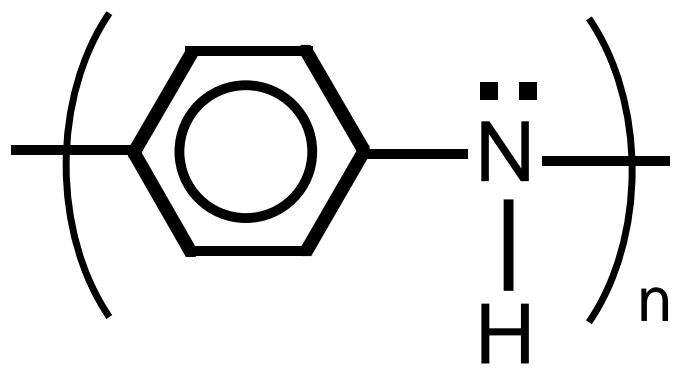

